\title{
Gravitational Waves and Time-Domain Astronomy
}

\author{
Joan Centrella ${ }^{1}$, Samaya Nissanke ${ }^{2}$ \& Roy Williams ${ }^{2}$ \\ ${ }^{1}$ NASA Goddard Spaceflight Center, Greenbelt, MD 20771, USA \\ email: Joan.Centrella@nasa.gov \\ ${ }^{2}$ California Institute of Technology, Pasadena, CA 91125, USA
}

\begin{abstract}
The gravitational-wave window onto the universe will open in roughly five years, when Advanced LIGO and Virgo achieve the first detections of high-frequency gravitational waves, most likely coming from compact binary mergers. Electromagnetic follow-up of these triggers, using radio, optical, and high energy telescopes, promises exciting opportunities in multimessenger time-domain astronomy. In the decade, space-based observations of low-frequency gravitational waves from massive black hole mergers, and their electromagnetic counterparts, will open up further vistas for discovery. This two-part workshop featured brief presentations and stimulating discussions on the challenges and opportunities presented by gravitational-wave astronomy. Highlights from the workshop, with the emphasis on strategies for electromagnetic follow-up, are presented in this report.
\end{abstract}

Keywords. gravitational waves, compact binaries, time domain astronomy, multi-messenger astronomy

\section{New Cosmic Messengers}

Gravitational waves (GWs) are a new type of cosmic messenger, bringing direct information about the properties and dynamics of sources such as compact object mergers and stellar collapse. The observable GW spectrum spans over 18 orders of magnitude in frequency, ranging from phenomena generated in the earliest moments of the Universe to vibrations of stellar-mass black holes (BHs).

For time-domain astronomy, two GW frequency bands stand out as being especially promising. The high frequency band, $\sim 1-10^{4} \mathrm{~Hz}$, will be opened by ground-based interferometric detectors starting around mid-decade. The strongest sources in that band are expected to be the mergers of stellar-mass compact objects, primarily $\mathrm{BH}$ binaries, neutron star (NS) binaries, and BH-NS binaries in the local universe out to several hundred Mpc. The low frequency band, $\sim 10^{-4}-10^{-1} \mathrm{~Hz}$, will be opened by space-based detectors in the 2020s. Merging massive black-hole (MBH) binaries, with masses in the range $\sim 10^{3}-10^{7} M_{\odot}$ and detectable out to high redshifts $(z>10)$, are expected to be the strongest sources here and the ones of greatest interest to time-domain astronomy. Other low-frequency sources include inspirals of compact objects into central MBHs in galaxies and compact stellar binaries with periods of tens of minutes to hourst.

The GWs emitted by binaries typically evolve upwards in frequency with time. When the binary components are well separated and spiralling together because of GW emission, the waveform is a sinusoid increasing in frequency and amplitude, also called a chirp.

$\dagger$ The very low frequency band, $\sim 10^{-9}-10^{-6} \mathrm{~Hz}$, will be opened by pulsar timing arrays later this decade. The most likely sources in this region are binaries containing MBHs of $\sim 10^{9} M_{\odot}$. 
The final merger, in which the binary components coalesce, produces a burst of radiation; that is followed by a ringdown phase in which the merged remnant typically settles down to an equilibrium configuration. The time-scales for those phases depend primarily on the masses of the binary components. For compact remnants, the inspiral might be detectable for about a minute, while the merger and ringdown occur on time-scales of roughly a few to tens of milliseconds. The detailed nature of the merging objects, for example moduli of the neutron star crust, is revealed in those last few milliseconds. For $\mathrm{MBH}$ binaries, space-based detectors should be able to observe the inspiral for several months. The ensuing merger and ringdown then occur over minutes to hours.

The possibility of electromagnetic (EM) counterparts of these GW sources raises exciting prospects for multi- messenger astronomy in the time domain. An EM counterpart greatly boosts confidence in a GW detection (Kochanek 1993). EM counterparts may be a precursor (possibly associated with the binary inspiral), a flash (triggered during the merger and/or ringdown phases), or an afterglow. The timescales for these signals depend on emissions from gas in the vicinity of the binary and can vary widely depending on the type of EM emission produced. Afterglows in particular can be very long-lived.

The opportunities for GW-EM multi-messenger time-domain astronomy generated lively and fruitful discussions during a two-session workshop held during the Symposium. This report presents the highlights from that workshop.

\section{High Frequency Gravitational Waves: Getting Ready for Detection}

The GW window onto the universe is expected to open around the middle of this decade, when the the first detections of GW signals from compact binaries are made by ground-based interferometers with kilometre-scale arms. Those first detections will mark the culmination of years of development by hundreds of scientists world-wide, and will inaugurate a new era in GW astronomy. This Section presents a status report on those efforts, and highlights important questions and challenges for this category of multimessenger time-domain astronomy.

\subsection{Status Update}

Currently there are three full-scale ground-based interferometric observatories: LIGO runs the 4-km observatories located in Hanford, Washington and Livingston, Louisiana, and the 3-km French-Italian Virgo detector located near Pisa, Italy. Both LIGO and Virgo were planned to be developed in stages. The initial detectors would be full-scale interferometers able to detect rare (nearby) events; the advanced detectors would be about a factor of 10 more sensitive, able to make multiple detections per year and to be true observational tools.

LIGO and Virgo have successfully reached their initial design sensitivities, completing several science data-taking runs in 2009-2010. According to current estimates of stellar-mass mergers, that many months of observation should have yielded a detection with probability $<2 \%$ (LSC 2010). Rather than waiting decades for a strong enough signal, LIGO and Virgo are undergoing upgrades to make joint detection rates at least yearly, perhaps weekly. Early science runs with Advanced LIGO/Virgo could start by mid-decade. As of September 2011, Virgo is still operating; the upgrade to Advanced Virgo is expected to follow the Advanced LIGO upgrade by about a year. Construction has also begun in Japan for the Large-Scale Cryogenic Gravitational-wave Telescope (LCGT; Kuroda 2010), an advanced detector that could start operating by the end of this decade. Another advanced detector may also be built in India. 
The initial LIGO and Virgo detectors have been used to carry out science runs, deriving upper limits on sources within their observational reach, and to develop data-analysis and detection strategies. An EM follow-up programme and a blind-injection test were exercised during the 2009-2010 science runs, providing valuable experience for the advanced detector era (and of particular significance for this workshop). The follow-up programme incorporated a prompt search for EM counterparts triggered by GW transients (LSC 2011c). Candidate GW events and their possible sky locations were identified using a low-latency analysis pipeline. The most promising sky positions for EM imaging were selected using a catalogue of nearly galaxies and Milky Way globular clusters. Within $\sim 30$ minutes, that directional information was sent to partner telescopes around the globe and also to the SwIFT gamma-ray satellite (see Fig. 1). Nine such events were followed up by at least one telescope (LSC 2011c).

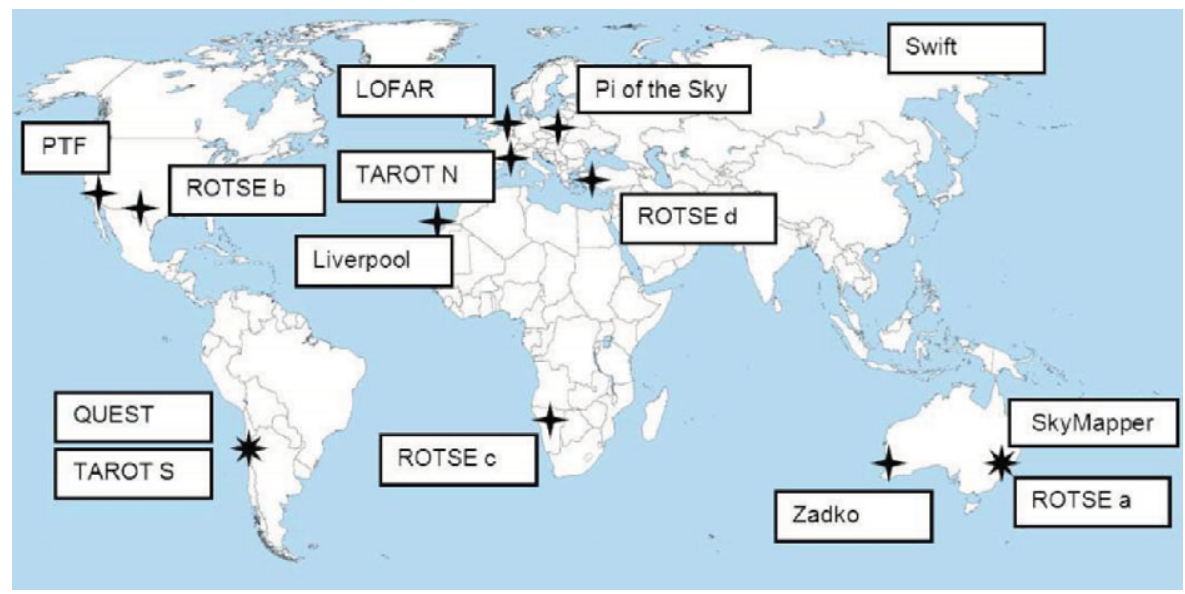

Figure 1. Telescopes that participated in the LIGO-Virgo EM follow-up exercise. Figure from LSC (2011c)

A blind injection test was also carried out during September 2010 (LSC 2011a). In this process - designed to provide a stress test for the full data analysis pipeline and science procedures - signals were secretly injected into the detector data stream by a small group of analysts. The parameters of those signals were sealed in an envelope, to be opened only after the entire collaboration had searched the data and carried out a full exercise of the processes, from potential detection to approval of a publication. In this case, a strong chirp signal was observed in the data shortly after injection. The data were vetted, sky positions were determined, and the GW trigger information was sent to several EM telescopes. As part of the process, LIGO and Virgo prepared a "data release" - a set of data from an injected signal, representing what could be released to the community when the first actual detections are made; see LSC (2011b).

\subsection{Expectations about $G W$ Source Detection}

GWs are produced by the dynamical motion of massive objects in space/time. Since they couple very weakly with matter, GWs are ideal cosmic messengers for bringing information from dark, hidden regions within galaxies. This section highlights some key expectations gleaned from studies of GW source detection, focusing on compact binary sources.

How many compact binaries are expected to be observed by advanced ground-based GW detectors? Rates for detection by Advanced LIGO and Virgo 
are estimated using projected detector sensitivities, plus compact binary merger rates derived from either the observed sample of galactic binary pulsars or population synthesis results (LSC 2010). A network consisting of three advanced detectors is expected to detect between $0.4-400 \mathrm{NS}-\mathrm{NS}$ binaries per year (the most realistic estimate being about 40 per year), out to a distance of $\sim 450 \mathrm{Mpc}$ for optimally oriented sources (that is, face-on and located directly above the detector). For stellar BH binaries, in which each $\mathrm{BH}$ has a mass $\sim 10 M_{\odot}$, the rates range from $0.2-300$ detections per year (most realistically $\sim 20$ per year), out to $\sim 2000 \mathrm{Mpc}$. For NS-BH binaries, where the BH has a mass $\sim 10 M_{\odot}$, the rates range from $0.2-300$ per year, the most realistic estimate being $\sim 10$ per year, out to $\sim 900 \mathrm{Mpc}$. Averaging over the sky and the source orientation reduces all those distance ranges by a factor of about two (Finn 1993).

What properties of these sources can be measured by observing the GWs emitted? GWs carry direct information about their sources. Broadly speaking, applying parameter estimation techniques to analyze the gravitational waveforms yields the binary masses, the spins and the orbital elements, as well as extrinsic parameters such as distance and position on the sky (Cutler 1994). It is hoped that the global GW network will eventually be able to elucidate deep secrets of extreme matter, ripped by space itself, through analysis of the merger waveform.

How well can these sources be localized on the sky? GW detectors are all-sky monitors. A single interferometer has a broad antenna pattern. It has poor directional sensitivity, but the localizations of sources on the sky can be refined by comparing the times of arrival of the signals in more than one detector. Using the network of three advanced detectors (Virgo plus the two LIGO sites) enables the sky positions to be limited to a region of $\sim 1-100 \mathrm{deg}^{2}$ (Fairhurst 2011, Wen 2010, Nissanke 2011). Adding additional interferometers, in particular one located out of the LIGO-Virgo plane such as LIGO India, brings improvements of factors $\sim 3$ (Schutz 2011). Note that the search regions are irregularly shaped and can have non-contiguous "islands" if the signals are near threshold (see Fig. 2), as is to be expected for many LIGO-Virgo sources.

What types of information can astronomers expect to receive from $\mathrm{GW}$ observatories? The advanced detector network will be able to supply the signal time of arrival and sky location of the source, along with an estimate of the false alarm rate (FAR). For merging compact binaries, the masses and spins of the components along with the inclination and luminosity distance will also be available. Other information may also be released: see LSC (2011b) and Fig. 2 for an example.

When will LIGO release to the public its rapid alerts for afterglow observers? Rapid release of triggers ( minutes) will begin under one of three criteria, according to the LIGO Data Management Plan (Anderson 2011): (a) after a GW detection has been confirmed and the collaboration agrees to begin the public programme, or (b) after a large volume of space-time has been searched by LIGO, or (c) if the detectors have been running for a long time. It is hoped that this release could be as early as 2016 . It should be noted, however, that these criteria may be changed in the future and the release date brought forward.

\subsection{Observing EM Counterparts: Challenges and Opportunities}

Mergers of NS-NS and NS-BH binaries are expected to generate EM radiation in several wavelength bands. Coupling observations of EM radiation and GWs from those sources opens up exciting new avenues for exploration. Since many GW detections may be near threshold, the identification of an EM counterpart would provide additional confirmation of the event. Direct measurements of the binary properties through GW observations will allow testing and deeper understanding of the underlying astrophysical models which are 


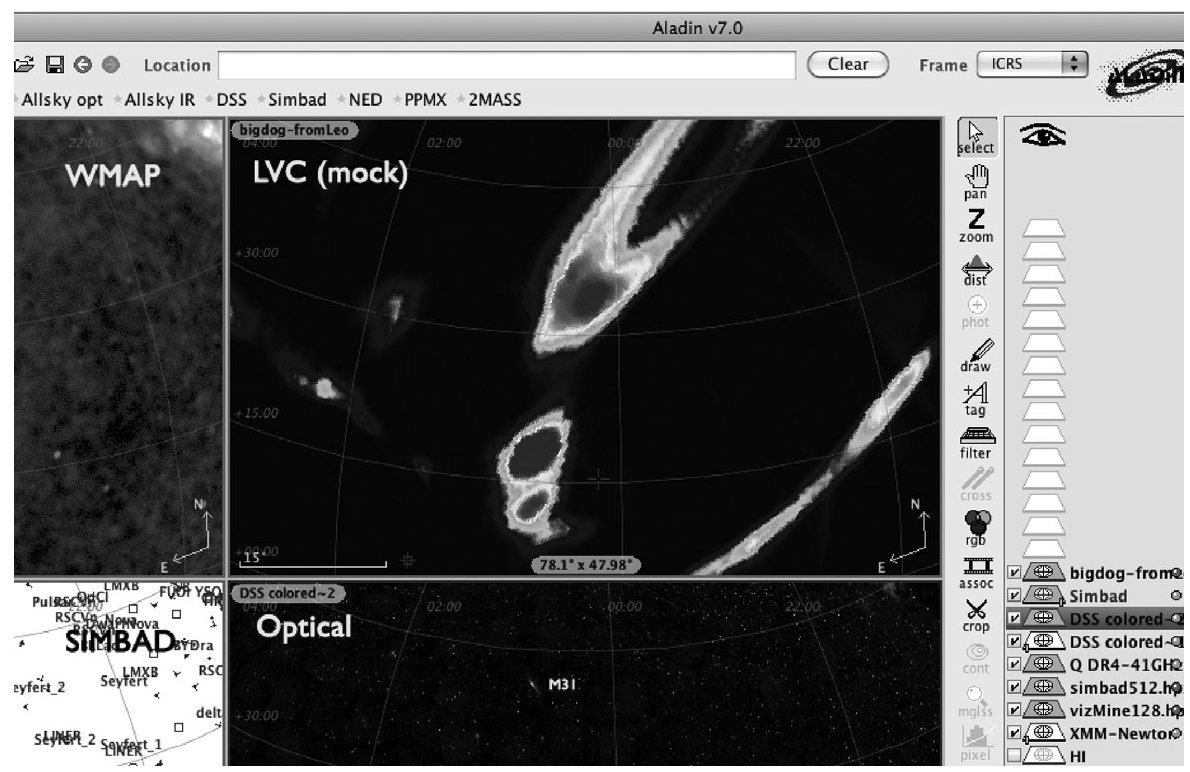

Figure 2. Non-contiguous search islands, illustrated with the Aladin "astronomical information system" (Fernique 2011). By converting the skymap to Healpix/FITS format, it can be compared directly with other astronmical images and catalogues. The panel 'LVC (mock)' corresponds to the skymap released as (LSC 2011b), with at least seven islands visible. Other panels show the same sky with other maps for comparison; left: WMAP data, lower-left: SIMBAD graduated catalog, and right: the Optical sky (DSS), where the galaxy M31 can be seen.

currently inferred only from EM radiation. This section captures exciting and challenging issues in this arena.

Which astrophysical phenomena generate promising EM counterparts to NS-NS and NS-BH mergers? These mergers are expected to produce collimated jets, observed near the axis as short-duration gamma-ray bursts (SGRBs). Afterglows from interactions of a jet with gas around the burst can be observed in the optical (near the axis, on time-scales of hours to days) and radio (isotropically, over weeks to years). A "kilonova" may also be produced from radioactive decay of heavy elements synthesized in the ejecta, yielding weak optical afterglows lasting several days ( $\mathrm{Li} \mathrm{1998).} \mathrm{Note} \mathrm{that}$ SGRBs are rare within the distance observable by Advanced LIGO and Virgo. Thus the isotropic emissions, particularly those from kilonovæ, are likely to be the most promising observable EM counterparts; see Metzger (2011) for details.

What information will astronomers want from the GW observatories? Most astronomers will first want the time and sky location of the event in order to plan the follow-up. Information about the binary components and orbits will also be valuable for understanding the underlying astrophysics. A smaller group of scientists may also want the gravitational-wave time-series information. A means for distributing that information is currently being worked out.

Which astronomical instruments will be especially useful in searching for EM counterparts? Satellites such as SWIFT and FERMI are needed to find GRBs, which are expected to be coincident with GW detections but only if the axis of the inspiral system points to the Earth ("beaming"). Other gamma or X-ray satellite observatories, current and planned, include MAXI, SVOM, and AstroSat. Rapid follow-ups by groundbased optical telescopes with wide fields of view (such as PTF-2, Pan-STARRS and LSST) and radio telescopes (LOFAR, ASKAP, EVLA, etc.) will search for the afterglows. 
Which EM follow-up strategies will produce the best astrophysical results? In the simplest scheme, all follow-up telescopes operate independently, pointing at the regions identified as having the greatest probability of containing the target. Since the source error regions on the sky from GW events will be relatively large irregularlyshaped ones that may have non-contiguous islands, many EM counterparts, particularly afterglows of relatively short duration, may be missed by this approach. Coordinated EM follow-ups, in which many telescopes operate co-operatively to cover the source region, could increase dramatically the odds of imaging successfully an EM counterpart (Singer 2011).

Would it be possible to observe the immediate optical "flash" that is expected within seconds of the merger time? There are all-sky optical monitors in operation and in the planning. The Pi Of The Sky observatory (Sokolski 2011) can locate a very fast transient to within seconds, down to magnitudes fainter than 12 . The planned global monitoring system from Los Alamos and LCOGT (Wren et al. 2010) will have the advantage of a much higher duty cycle, because of its distributed nature and multiple telescopes at each site.

How early a notice of a GW trigger is useful, and possible? Since early notification increases not only the odds of imaging successfully the counterpart but also raises the amount and types of information that will be gained, notices should be sent as early as possible. In the recent follow-up program carried out by LIGO and Virgo, notices were sent out within 30 minutes of detection (LSC 2011c), and efforts are continuing to reduce that to 1 minute. Detecting a GW signal during a binary inspiral (before the merger) and releasing the information before the burst occurs is even more interestingand challenging; Cannon (2011) provides an early study.

Which triggers should be followed up? How low a significance is tolerable? Strong, nearby GW sources should have a low false-alarm rate (FAR) and produce more robust data, including more accurate values for the binary parameters and sky location. However, signals near threshold might be more common. The answers to those questions will depend on the actual detection rate.

What more information about the local universe is needed in order ro prepare for the data from Advanced LIGO and Virgo? Two items in particular are needed: a complete publicly-available catalogue of nearby galaxies (White 2011, Kulkarni 2009), and an inventory of known transients within reach of Advanced LIGO and Virgo. For faint transients, it may be that the lack of such a catalogue, and the large numbers of sources, can make it very difficult to identify a GW afterglow even if there is a wide, deep telescope available. By using spatial coincidences with a complete galaxy catalogue for those events within $200 \mathrm{Mpc}$, the number of false-positve transients in a typical GW error box of $\sim 10 \mathrm{deg}^{2}$ is reduced by three orders of magnitude (Kulkarni 2009).

\section{Low-Frequency Gravitational Waves: Looking to the Future}

The low-frequency window contains a wealth of astrophysical sources. Because of noise from fluctuating gravity gradients in the Earth at frequencies below a few $\mathrm{Hz}$, lowfrequency GWs can only be observed with space-based detectors. The most highly developed proposal is the Laser Interferometer Space Antenna (LISA), which consists of three satellites orbiting the Earth in a triangular configuration with arm lengths $\sim 5 \times 10^{6} \mathrm{~km}$ (Jennrich 2009). Detectors such as LISA will observe coalescing MBH binaries inspiralling over a period of several months, followed by the final merger and ringdown, as well as inspirals of compact objects into central MBHs in galaxies and compact stellar binaries with periods of minutes to hours. 
What is the current status of low frequency GW detectors? Owing to budgetary problems, ESA and NASA terminated their partnership to develop LISA in the spring of 2011, and both agencies are now looking at lower-cost concepts. On the ESA side, studies are underway for the New Gravitational-wave Observatory (NGO, also known informally as LISA-lite, EuLISA, and eLISA), which is similar to LISA but with shorter arm lengths of $\sim 1 \times 10^{6} \mathrm{~km}$. In the US, NASA is also examining concepts for a Space Gravitational-wave Observatory (SGO).

What can be learned from observing low-frequency GWs from MBH mergers? These space-based interferometers can observe MBH mergers (Sesana 2011) over a period of several months at relatively high signal-to-noise, allowing precision measurements of the binary properties, plus sky localization to $<100 \mathrm{deg}^{2}$. The expected merger time can be predicted and broadcast weeks or months in advance, providing excellent opportunities for EM follow-ups. The rates for $\mathrm{MBH}$ mergers are expected to be at least several per year, with the actual values depending on the instrument sensitivity.

What are the prospects for EM counterparts of MBH mergers? MBH mergers are astrophysically rich systems, with a variety of possible EM signals as precursors, flashes and afterglows (Schnittman 2010). Since MBH mergers are considered central to our understanding of galaxy and $\mathrm{MBH}$ assembly history and demography as well as galaxy-MBH co-evolution, the astrophysical payoffs will be significant (Komossa 2003).

\section{Summary}

The GW window onto the universe will open this decade, when Advanced LIGO and Virgo make the first detections of high-frequency GW signals that are expected to come from merging compact binaries. Strategies for EM follow-ups of the GW triggers are being designed and tested to search for radio, optical and high-energy counterparts. Searches for coincident GW and high-energy neutrinos will be made in co-ordination with the IceCube project (Bartos 2011). Co-ordinated searches, coupled with complete catalogues of galaxies and transients in the local universe, are needed in order to maximize the science from these multi-messenger studies. In the next decade, space-based interferometers will open the low-frequency window with observations of merging $\mathrm{MBH}$ binaries. These observations, and their EM counterparts, will provide important information on the evolution of structure and MBHs over cosmic time.

\section{Acknowledgements}

It is a pleasure to thank Eric Chassande-Mottin, Ed Daw, Stephen Fairhurst, Jonathan Gair, Mansi Kasliwal, Stefanie Komossa, Brian Metzger, Larry Price, Jeremy Schnittman, Alberto Sesana, and Leo Singer, who delivered excellent short presentations at the GW workshops that stimulated interesting and important discussions.

\section{References}

LSC 2010: Abadie, J., et al. 2010, LIGO Scientific Collaboration and Virgo Collaboration, Class. Quant. Grav., 27, 173001

LSC 2009: Abbott, B. P., et al. 2009, LIGO Scientific Collaboration and Virgo Collaboration, Reports on Progress in Physics, 72, 076901

LSC 2011c: Abbott, B. P., et al. 2011c, LIGO Scientific Collaboration and Virgo Collaboration, http://arxiv.org/abs/1109.3498

Anderson, S \& Williams, R.D. LIGO Data Management Plan https://dcc.ligo.org/cgi-bin/DocDB/RetrieveFile?docid=9967

Apostolatos, T., Kennefick, D., Ori, A., \& Poisson, E. 1993, Phys.Rev., D47, 5376 
Bartos, I., Finley, C., Corsi, A., \& Marka. S. 2011, http://arxiv.org/abs/1108.3001

Cannon, K., et al. 2011, submitted

Cutler, C. \& Flanagan, E. 1994, Phys. Rev., D49:2658

Fairhurst, S. 2011, Class. Quantum Grav., 28, 105021

Fernique, P., Boch, T., et al. 2011, http://aladin.u-strasbg.fr/

Finn, L. S. \& Chernoff, D. F. 1993, Phys. Rev. D, 47:2198

Harry, G. M., et al. 2010, Class.Quant.Grav., 27:084006, 2010.

Jennrich, O. 2009, LISA technology and instrumentation. Class. Quant. Grav., 26:153001, 2009.

Kochanek, C. S., and Piran, T. 199 ApJ, 417, L17

Komossa, S. 2003, in: J. M. Centralla (ed.), The Astrophysics of Gravitational Wave Sources, AIP Conf. No. 686, (Berlin: Springer), p. 161

Kulkarni, S., \& Kasliwal, M.M. Proc. RIKEN Symp., Astrophysics with All-Sky X-Ray Observations, 312, arXiv:0903.0218

Kuroda, K., The LCGT Collaboration. 2010, Class. Quant. Grav., 27, 84004

Li, L.-X. \& Paczyński, B. 1998, ApJ, 507, L59

LSC 2011a: LIGO Scientific Collaboration and Virgo Collaboration, 2011a, http://www . ligo.org/news/blind-injection.php

LSC 2011b: LIGO Scientific Collaboration and Virgo Collaboration. 2011b, Mock data release GW100916 Colloq. 'Big Dog Data' http://www.ligo.org/science/GW100916

Metzger, B. D. \& Berger, E. 2011, http://arxiv.org/abs/1108.6056

Samaya, M., Nissanke, J. L., Sievers, N. D., \& Holz, D. E. 2011, ApJ, 739, 99

Schnittman. J. D. 2011, Class. Quant. Grav., 28, 94021

Schutz, B. F. 2011, Class. Quant. Grav., 28, 125023

Sesana, A., Gair, J., Berti, E., \& Volonteri, M. 2010, Phys. Rev., D83, 044036, 2011.

Singer, L., Price, L., \& Speranza. A., https://dcc.ligo.org/cgi-bin/DocDB/ShowDocument? docid $=$ G1100983

Sokolowski, M. http://grb.fuw.edu.pl/

Wen, L. \& Chen, Y., Phys. Rev., D81:082001, 2010.

White, D. J., Daw, E. J., \& Dhillon, V. S. 2011, Class. Quant. Grav., 28, 85016

Wren, J., Vestrand, W. T., Wozniak, P., \& and Davis, H. 2010, SPIE Proc., 7737, 773723 